\title{
MODERN STATE OF CRUSTAL STRESSES OF THE APENNINE PENINSUlA AND ADJACENT TERRITORIES (CENTRAL MEDITERRANEAN REGION)
}

\author{
P. A. Savvichev, Yu. L. Rebetsky \\ O.Yu. Schmidt Institute of Physics of the Earth of RAS, Moscow, Russia
}

\begin{abstract}
This paper discusses models showing the formation of the Central Mediterranean region and the geodynamic setting of the Apennine Peninsula. Cataclastic analysis is used for a repeated reconstruction of the Central Mediterranean region. The catalogue of earthquake focal mechanisms includes 662 events $\left(3.6 \leq \mathrm{M}_{\mathrm{b}} \leq 6.5\right)$ recorded in the study area from 1977 to 2015 (Global CMT, http://www.globalcmt.org; RCMT, http://rcmt2.bo.ingv.it/index.html; Italian CMT dataset, http://rcmt2.bo.ingv.it/Italydataset.html). The reconstruction yielded the directions of principal stresses (including algebraically maximum and minimum ones), locations of domains differing in geodynamic regime, Lode - Nadai coefficients, and orientation of tangential shear stresses acting from the mantle to the crust. By comparing our results to the published data obtained by M.-L. Zoback's method, we have identified differences in the orientations of maximum horizontal compression axes at points where the stress ellipsoid takes on its critical values. It is revealed that the strongest earthquakes $(M>6)$ were generated in the areas characterized by the minimum and average relative stress magnitudes.
\end{abstract}

Key words: tectonophysics; stress; earthquake source; mechanism; geodynamics

For citation: Savvichev P.A., Rebetsky Yu.L., 2019. Modern state of crustal stresses of the Apennine Peninsula and adjacent territories (Central Mediterranean region). Geodynamics \& Tectonophysics 10 (4), 921-935. doi:10.5800/GT-2019-10-4-0449.

Funding: This work was performed as part of the State order of O.Yu. Schmidt Institute of Physics of the Earth of RAS. 


\title{
СОВРЕМЕННОЕ НАПРЯЖЕННОЕ СОСТОЯНИЕ КОРЫ АПЕННИНСКОГО ПОЛУОСТРОВА И СОПРЕДЕЛЬНЫХ ТЕРРИТОРИЙ (ЦЕНТРАЛЬНОЕ СРЕДИЗЕМНОМОРЬЕ)
}

\author{
П. А. Саввичев, Ю. Л. Ребецкий
}

Институт физики Земли им. О.Ю. Шмидта РАН, Москва, Россия

\begin{abstract}
Аннотация: В данной работе рассматриваются возможные модели образования Центрального Средиземноморья и геодинамическая обстановка Апеннинского полуострова. С помощью метода катакластического анализа проводится повторная реконструкция Центрального Средиземноморья. Каталог механизмов очагов землетрясений включает в себя данные Global CMT (http://www.globalcmt.org), RCMT (http://rcmt2.bo.ingv.it/index.html) и Итальянской базы данных (Italian CMT dataset) (http://rcmt2.bo.ingv.it/Italydataset.html). Каталог составили 662 события с магнитудами $3.6 \leq \mathrm{M}_{\mathrm{b}} \leq 6.5$, произошедшие за период с 1977 по 2015 г. Результатом реконструкции является ориентация направлений алгебраически максимальных и минимальных главных напряжений, расположение доменов геодинамического режима и коэффициента Лоде - Надаи, а также ориентация поддвиговых касательных напряжений, действующих со стороны мантии на кору. Проводится сравнение с уже имеющимися данными, которые были получены с помощью методики М.Л. Зобак, найдены отличия в ориентации осей наибольшего горизонтального сжатия в местах, где вид эллипсоида напряжений принимает свои критические значения. По данным об относительных величинах напряжений показано, что формирование наиболее сильных событий $(M>6)$ происходило в областях с минимальными и средними относительными величинами.
\end{abstract}

Ключевые слова: тектонофизика; напряжение; очаг; механизм; геодинамика

\section{1. ВВЕДЕНИЕ}

Исследуемый регион коры Апеннинского полуострова и ближайшего окружения представляет собой западную часть Альпийско-Гималайской тектонической системы, образованной в течение последних 100 млн лет в результате коллизии между крупными тектоническими плитами, такими как Африка, Арабия и Евразия, а также с участием других, второстепенных, более мелких плит - Адрия, Анатолия, Иберия. Тирренейский бассейн и Апеннинский горный пояс сформированы в рамках геодинамической структуры в течение 35 млн лет.

За последние десятилетия были предложены несколько моделей для объяснения данной проблемы в системе Тиррен-Апеннин. Первая модель рассматривает отступ Африканской плиты, ответственной за пластическую/жесткую экструзию континентального материала на восток [Tapponnier, 1977; Boccaletti et al., 1982; Mantovani et al., 1993]: Тирренское расширение развивалось перпендикулярно объемному сокращению, вызванному латерально продвигающейся Африканской плитой, окруженной фронтом сжатия дуги Калабрии. В рамках этой модели нагружения была предпринята попытка объяснить режим горизонтального растяжения в коре Апеннин.

Вторая модель рассматривает погружение - субдукцию Африканской плиты - как движущий механизм [Malinverno, Ryan, 1986; Royden et al., 1987; Patacca, Scandone, 1989; Patacca et al., 1990; Royden 1993], создающий задуговое расширение в Тирренской области и приращение в Апеннинской цепи. В этой модели миграция доменов сжатия и растяжения на восток обусловлена откатом отступающей зоны субдукции (механизм ролл-бэк): изгиб Калабрийской дуги произошел вследствие высокой скорости отступления субдуктивного ионического домена [Malinverno, Ryan, 1986].

Третья модель рассматривает выделение потенциальной энергии, хранящейся в поднимающемся после коллизии клине альпийской коры. Результатом этого процесса является деформационное расширение. Тирренское растяжение могло быстро развиваться в ранее застывшей шовной зоне альпийского столкновения мела - палеогена, окруженного зоной сжатия [Reutter et al., 1980; Horvath, Berckhemer, 1982; Channell, 1986]. С точки зрения механики разрушение литосферы может быть объяснено удалением мантийной литосферы: этот процесс провоцирует подъем и, в свою очередь, ее разрушение, 
если не обеспечивается поддержка окружающей литосферой.

Эти три модели основаны на трех различных механизмах деформации: субдукционном сжатии, вызванном сближением Африки и Европы; процессе отката слэба (slab rollback) - Африканской плиты и гравитационном коллапсе. Предполагается возможность объединения этих трех деформационных механизмов [Faccenna et al., 1996].

Сегодняшние модели деформации Центрального Средиземноморья очень неоднородны, в них присутствуют тектонические структуры растяжения и сжатия, сосуществующие в довольно ограниченном пространстве одновременно. Такая существенная пространственная неоднородность напряженного состояния подсказывает исследователю, что механизм деформирования коры региона труднообъясним в рамках простых (одномерных) условий нагружения.

\section{2. ИСТОРИЯ ИЗУЧЕНИЯ НАПРЯЖЕНИЙ АПЕННИНСКОГО ПОЛУОСТРОВА}

Исследуемый регион является сейсмически активным, наиболее часто события происходят в коре центральной части Апеннин, в зоне субдукции у о. Сицилия, в долине реки По. Актуальность изучения напряженного состояния земной коры обусловлена серией достаточно сильных землетрясений, произошедших в центральной части Италии (Норча, 2016 г., $M_{\mathrm{w}}=6.3$; Л’Акуила, 2009 г., $M_{\mathrm{w}}=6.3$ и др.). Заметим, что даже события со средней магнитудой могут нанести значительный урон, так как исследуемый регион достаточно густонаселенный, а существующие строения, имеющие большую историческую значимость, не удовлетворяют современным требованиям безопасности. Знания о фокальных механизмах землетрясений и о напряжениях, вызывающих эти землетрясения, позволяют понять, какие механизмы нагружения коры на сегодняшний день являются наиболее активными [Rebetsky, 2003]. Изучение генезиса напряжений является важной фундаментальной проблемой геодинамики. Данные о напряжениях могут быть использованы для тектонофизического районирования разломов и поэтому важны с позиции оценки сейсмической опасности [Rebetsky, Kuzikov, 2016].

Новый этап в реконструкции современных напряжений коры Европы связан с работами М.Л. Зобак и ее коллег [Zoback et al., 1989; Zoback, 1992] в рамках проекта World Stress Map (WSM). Дальнейшее развитие этих исследований было выполнено в работах [Heidbach et al., 2010, 2016]. Основным результатом этих исследований являются карты пространственного распределения осей напряжений наибольшего горизонтального сжатия, составленные на основании данных о механизмах очагов единичных землетрясений [Zoback et al., 1989] или их групп [Zoback, 1992].

Для коры территории Италии и ближайшего окружения данные WSM [Heidbach et al., 2016] показывают определенную вариабельность (рис. 1) ориентации осей наибольшего горизонтального сжатия, которая может быть связана как с особенностями тектоники и неоднородным по площади режимом деформирования, так и с разнородностью исходных данных, использованных для инверсии напряжений. Так, скважинные данные, данные выбуривания кернов и им подобные, полученные для глубин менее 3 км, имеют масштаб усреднения напряжений в первые метры. Геологические данные о деформациях структур, имеющие масштаб усреднения от первых до сотен метров и километров, относятся к приповерхностным объектам, которые в прошлом могли быть на больших глубинах. Наконец, сейсмологические данные о механизмах очагов землетрясений в основном относятся к глубинам 520 км. Поскольку согласно методике обозначенных исследований [Zoback, 1992] для инверсии напряжений качества А и Б требуется использовать данные о 3-4 землетрясениях с магнитудами не менее 3.0-4.5, то, как следует из работы [Heidbach et al., 2010], масштаб усреднения для исследуемой территории был не менее 100 км.

Таким образом, проект WSM продемонстрировал существование поля напряжений первого порядка (масштаб тектонических плит), возникающего благодаря движению плит, а также существование поля напряжений второго порядка (регионального), которое зависит в основном от внутриплитовых напряжений [Müller et al., 1992; Zoback, 1992; Hillis, Reynolds, 2000; Tingay et al., 2010]. В районах с высокой сейсмической активностью может быть выделено также напряжение третьего порядка (локальное), которое связывают с наличием активных разломов, локальных неоднородностей, контрастов плотностей и др. [Bell, 1996; Yale, 2003; Tingay et al., 2005]. Данные о локальных напряжениях также могут быть получены по сейсмическим записям локальных сетей.

Такая иерархичность напряжений, связанная с типом исходных данных, может объяснить различные повороты осей главных напряжений [Mariucci et al., 2002; Carminati et al., 2010], что и наблюдалось в зонах разломов, активных разломах или механически слабых или хрупких породах. Знание современного напряженного состояния в тектонически активных регионах важно также для понимания механики различных геолого-геофизических процессов, включая разломы и землетрясения [Zoback, 1992; Sassi, Faure, 1996; Suppe, 2007]. 


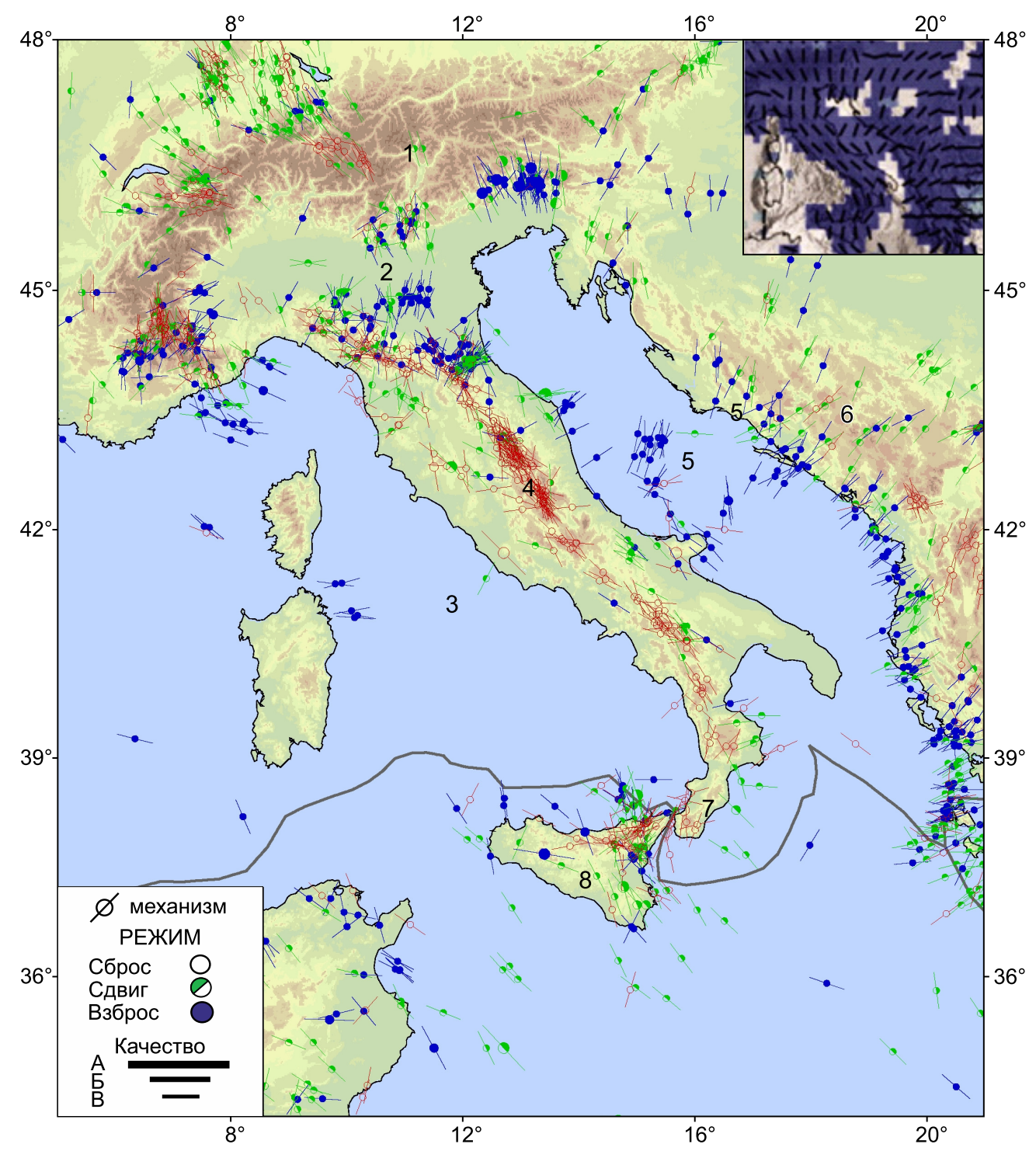

Рис. 1. Данные об ориентации осей наибольшего горизонтального сжатия для земной коры Апеннинского полуострова и сопредельных районов из проекта WSM 2016 и работы [Heidbach et al., 2010] (вверху справа).

Fig. 1. Orientations of the axes of maximum horizontal compression of the crust in the Apennine Peninsula and adjacent regions, according to WSM 2016 Project and [Heidbach et al., 2010] (top right).

\section{3. О МЕТОДИЧЕСКИХ ПРОБЛЕМАХ ИНВЕРСИИ НАПРЯЖЕНИЙ}

Как выше уже было отмечено, на картах WSM представлены данные об ориентации осей наибольшего горизонтального сжатия $\sigma_{H}$. Эти данные в картах WSM получены либо прямыми измерениями полного тензора напряжений в шахтах и скважинах, либо из данных о механизмах очагов землетрясений в виде ориентации осей главных напряжений $\sigma_{i}(i=1,2,3)$, задаваемых тремя углами Эйлера. Именно при интерпретации сейсмологических данных возникает первая проблема методики М.Л. Зобак [Zoback et al., 1989; Coblentz, Richardson, 1995; Heidbach et al., 2007, 2008, 2010].
В методе М.Л. Зобак ориентация осей напряжений наибольшего горизонтального сжатия получается как проекция максимального сжимающего напряжения $\sigma_{3}$ на горизонтальную плоскость. Но на самом деле для определения ориентации осей наибольшего горизонтального сжатия недостаточно данных об углах Эйлера. Ниже представлены выражения для определения напряжений $\left(\sigma_{n n}\right)$ в произвольном горизонтальном направлении $n$ :

$$
\sigma_{n n}=-p+\tau\left[\begin{array}{c}
\left(1-\frac{1}{3} \mu_{\sigma}\right) l_{1 n}^{2}+ \\
+\frac{2}{3} \mu_{\sigma} l_{2 n}^{2}-\left(1+\frac{1}{3} \mu_{\sigma}\right) l_{3 n}^{2}
\end{array}\right],
$$

где $l_{\text {in }}$ - направляющие косинусы осей главных напряжений $\sigma_{i}(i=1,3)$ в топоцентрической системе 

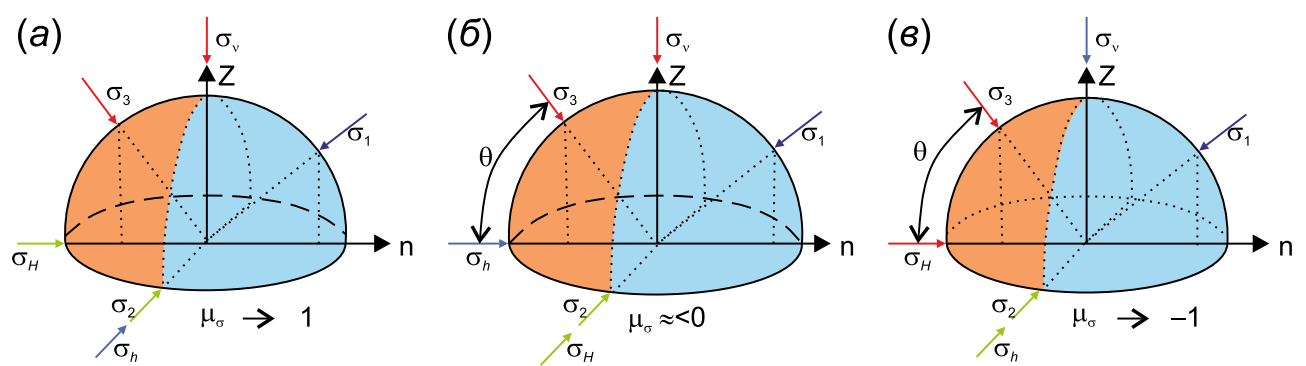

Рис. 2. Изменение направления действия наибольшего горизонтального сжатия при изменении формы эллипсоида напряжений (значения коэффициента $\mu_{\sigma}$ ) при постоянной ориентации осей главных напряжений: $(a)-\mu_{\sigma} \rightarrow 1,(\sigma)$ $-\mu_{\sigma} \approx<0,(8)-\mu_{\sigma} \rightarrow-1 . \mathrm{Z}$ - направление на зенит, $n-$ направление в горизонтальной плоскости, лежащее также в плоскости действия двух главных напряжений $\sigma_{1}$ и $\sigma_{3}$.

Fig. 2. Changes in the direction of maximum horizontal compression in case of changes in the shape of the stress ellipsoid (values of coefficient $\mu_{\sigma}$ ) with constant orientations of the principal stress axes: $(a)-\mu_{\sigma} \rightarrow 1,(\sigma)-\mu_{\sigma} \approx<0,(8)-\mu_{\sigma} \rightarrow-1$. $\mathrm{Z}$ - zenith direction; $\mathrm{n}$ - direction in the horizontal plane, which also lies in the plane of the two principal stresses $\sigma_{1}$ and $\sigma_{3}$.

координат, значения которых определяются выражениями:

$$
\begin{aligned}
& \sigma_{1}=-p+\tau\left(1-\frac{1}{3} \mu_{\sigma}\right), \\
& \sigma_{1}=-p+\frac{2}{3} \mu_{\sigma} \tau, \\
& \sigma_{3}=-p-\tau\left(1+\frac{1}{3} \mu_{\sigma}\right) .
\end{aligned}
$$

Здесь $\tau$ и $p$ - соответственно максимальное касательное напряжение и изотропное давление. Как следует из (1) и (2), девиаторная часть напряжений зависит не только от углов Эйлера, но и от вида тензора напряжений, определяемого коэффициентом Лоде-Надаи $\left(-1 \leq \mu_{\sigma} \leq 1\right)$. Отметим, что в нашей работе правило знаков для нормальных напряжений такое, как и в механике сплошной среды, т.е. растяжение положительно. В этой связи $\sigma_{1}-$ главное напряжение минимального сжатия, а $\sigma_{3}-$ главное напряжение максимального сжатия.

Выражения (1) и (2) позволяют рассмотреть простой пример, в котором ось промежуточного главного напряжения $\left(\sigma_{2}\right)$ строго горизонтальна $l_{2 n}=0$ (рис. 2), а оси двух других главных напряжений имеют углы погружения, близкие к $45^{\circ}$ (режим вертикального сдвига). В случае, если вид тензора напряжений изменяется от чистого сдвига $\left(\mu_{\sigma}<0\right)$ до одноосного растяжения $\left(\mu_{\sigma}=-1\right)$, ось наибольшего горизонтального сжатия $\sigma_{H}$ будет совпадать с $\sigma_{2}$. При чистом сдвиге $\sigma_{H}=\sigma_{h}$. Если вид тензора напряжений отвечает одноосному сжатию $\left(\mu_{\sigma}=1\right)$, с направлением промежуточного главного напряжения будет совпадать ось напряжений меньшего горизонтального сжатия $\sigma_{h}$.

Таким образом, существует ограничение на использование правила о том, что горизонтальная проекция оси максимального сжатия определяет ориентацию напряжения наибольшего горизон- тального сжатия $\sigma_{H}$. В диапазоне значений коэффициента $0 \leq \mu_{\sigma} \leq 1$, т.е. для вида тензора от одноосного сжатия до чистого сдвига правило М.Л. Зобак работает. Но при $\mu_{\sigma}<0$ существует предельный угол $\theta$ погружения оси максимального сжатия $\sigma_{3}$, больше которого это правило не выполняется. Для случаев, рассмотренных на рис. 2, б, в, выполнение правила М.Л. Зобак требует $\cos \theta<-\frac{2 \mu_{\sigma}}{\left(3+\mu_{\sigma}\right)}$. Предельные значения этого угла для $\mu_{\sigma}=$ $=-0.5 ;-0.75 ;-0.9$ будут соответственно 66,48 и $31^{\circ}$.

Как показывают практические расчеты, режим напряженного состояния с субгоризонтальной ориентацией оси промежуточного главного напряжения $\sigma_{2}$ имеет место для активных континентальных окраин - в сейсмофокальных областях зон субдукции [Rebetskii, Marinin, 2006; Rebetsky, 2009; Rebetsky, Polets, 2014]. В коре внутриконтинентальных орогенов присутствуют достаточно обширные участки, для которых наблюдаются существенные отклонения от вида тензора напряжений чистого сдвига [Rebetsky et al., 2012, 2013; Rebetsky, Alekseev, 2014].

Одной из задач проекта является проверка результатов, полученных с использованием метода М.Л. Зобак, для коры Италии (см. рис. 1).

\section{4. ИСХОДНЫЕ СЕЙСМОЛОГИЧЕСКИЕ ДАННЫЕ}

Для реконструкции параметров современного поля напряжений в земной коре Италии и ближайшего окружения на основе Итальянской базы данных (Italian CMT dataset http://rcmt2.bo.ingv. it/Italydataset.html) был создан каталог механизмов очагов землетрясений (рис. 3) для области, по площади несколько превышающей исследуемый 


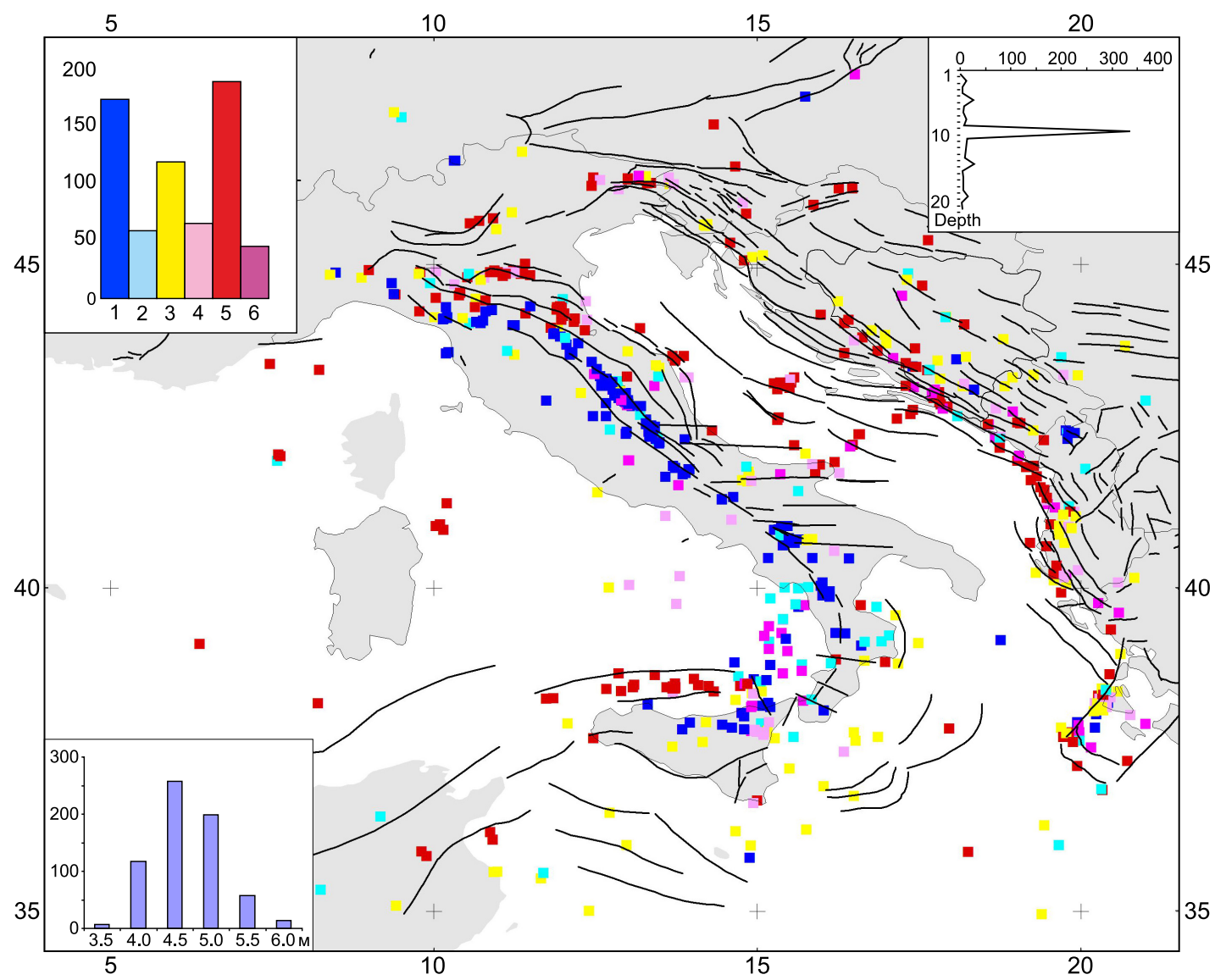

Рис. 3. Эпицентры землетрясений и кинематический тип механизма очагов землетрясений (1 - сбросы, 2 - сбрососдвиг, 3 - сдвиги, 4 - сдвиговзброс, 5 - взбросы, 6 - взрезы или поддвиги). Слева внизу представлена диаграмма распределений по магнитуде, справа сверху - распределение по глубине, а слева вверху - по кинематическому типу разрыва в очаге.

Fig. 3. Earthquake epicenters and the kinematic types of focal mechanisms (1 - normal faults, 2 - strike-slip fault with normal component, 3 - strike-slip faults, 4 - reverse fault with strike-slip component, 5 - reverse faults, 6 - incision or underthrust faults). Distribution diagrams: bottom left - magnitude; top right - depth; top left - kinematic types of faults in the earthquake sources.

регион (4-22 в.д. и 30-50 с.ш.). База данных включает в себя решения из каталогов Global CMT (http://www.globalcmt.org), RCMT (http://rcmt2.bo. ingv.it/index.html) и каталога Пондрелли [Pondrelli et al., 2006]. Необходимость некоторого расширения области реконструкции напряжений при создании каталога землетрясений связана с особенностями применяемой методики (см. далее).

Каталог составили 662 события с магнитудами $3.6 \leq \mathrm{M}_{\mathrm{b}} \leq 6.5$, произошедшие за период с 1977 по 2015 г. Анализ сейсмологических данных показал, что большая часть этих событий приходится на глубины до 20 км, и в основном они все расположены на глубине около 10 км (фиксированная глубина, которая принимается, когда данных недостаточно для точного определения глубины очага).

Механизмы с кинематическим типом сброса расположены преимущественно в коре Апеннин, вдоль линий разломов, в дуге Калабрии преоблада- ет смешанный тип: от взбросов и взрезов в Тирренском море рядом с о. Сицилия до сбросов на границе с сушей и сдвигов во внешней дуге Калабрии. В коре Динарид и в коре Адриатического моря преобладают взбросовые механизмы с небольшим количеством сдвиговых.

\section{5. МЕТОДИКА}

В нашей работе для получения данных о напряжениях используется метод катакластического анализа (МКА) разрывных смещений Ю.Л. Ребецкого [Rebetsky, 2003]. Этот метод, в отличие от метода М.Л. Зобак, не просто статистический, он основан на ряде положений теории хрупкого разрушения и пластичности. Главным отличительным моментом МКА является наличие в нем правил формирования выборки механизмов очагов землетрясений, по 
которой в дальнейшем будет производиться расчет напряжений.

По методу М.Л. Зобак такие выборки из 3-5 событий также создаются либо на основе наперед задаваемого окна усреднения, либо на основе требования минимального числа событий [Heidbach et al., 2010]. В зависимости от разброса в ориентации осей $P$ и $T$ механизмов очагов землетрясений в выборке, результатам расчетов присваивается определенный уровень качества.

Создание однородной выборки в МКА базируется на положениях механики о расчете необратимых деформаций. В окрестности каждой трещины (землетрясения) в результате относительного перемещения ее бортов формируется область упругой разгрузки, в пределах которой существенно изменяется напряженное состояние. В этой области происходит уменьшение накопленной внутренней упругой энергии и, следовательно, уменьшение интенсивности тензора средних по области упругих деформаций и напряжений. Линейный размер этой области может в десятки раз превышать линейный размер очага и зависит от состояния среды [Rebetsky, Lermontova, 2016]. Для создания начальной выборки землетрясений необходимо соблюдать пространственную близость очагов землетрясений. Суммирование остаточных деформаций производится только в пределах области взаимного пересечения объемов упругой разгрузки множества разрывов (землетрясений). В пределах этой области происходит суммирование деформаций, снимаемых в процессе землетрясений. Землетрясения из такой выборки называются начальной выборкой землетрясений. Начальная выборка структурно-кинематических данных о механизмах очагов землетрясения, сформированная фактически на основе пространственной близости при учете энергетической мощности событий, еще не может рассматриваться как финальная, характеризующая однородную фазу деформирования исследуемого объема.

Созданная на основе кумулятивного принципа (пересечение областей упругой разгрузки) начальная выборка землетрясений далее анализируется на однородность автоматизированными процедурами МКА. Под однородностью понимается определенная согласованность механизмов очагов землетрясений, включенных в нее. Эта согласованность механизмов проверяется на основе энергетических критериев однородности MКА [Rebetsky, 2003]. С физических позиций критерии однородности требуют наличия острого угла между направлением смещения на плоскости разрыва (очага) и касательным напряжением искомого напряженного состояния. Это означает, что для искомого напряженного состояния землетрясения приводят только к уменьшению упругой энергии. Фактически эти критерии требуют, чтобы вариабельность механизмов очагов землетрясений не была слишком большой.

Сама проверка на однородность механизмов очагов осуществляется последовательно для каждого землетрясения, вошедшего в начальную выборку. При этом землетрясения в выборке располагаются в порядке их возникновения, т.е. согласно шкале времени. Землетрясения, не прошедшие проверку на однородность, исключаются из дальнейшей обработки. Оставшиеся события формируют однородную выборку механизмов очагов землетрясений, по которой для выбранной точки литосферы определяются параметры напряжений.

В представленной работе реализованы два первых этапа алгоритма МКА, позволяющие определять в дополнение к параметрам эллипсоида напряжений (первый этап) также и нормированные величины максимальных касательных напряжений $(\tau)$ и эффективного давления $\left(p^{*}\right)$. Нормировка напряжений производится на величину прочности сцепления массивов, которая остается неизвестной и считается постоянной для региона.

Алгоритм определения нормированных напряжений на втором этапе МКА фактически опирается на факт увеличения разброса в ориентации механизмов очагов землетрясений при уменьшении интенсивности напряжений. Соответственно, области, где вариабильность механизмов уменьшается, определяют участки коры высокой интенсивности напряжений. Возможность получения подобных данных связана с анализом распределения на диаграмме Мора нормальных и касательных напряжений в очагах землетрясений из однородных выборок очагов землетрясений, сформированных на первом этапе МКА [Rebetsky, 2003]:

$$
\begin{aligned}
& \sigma_{n n}{ }^{*}=-p^{*}+\tau\left[\begin{array}{c}
\left(1-\mu_{\sigma}\right) l_{1 n}^{2}- \\
-\left(1+\mu_{\sigma}\right) l_{3 n}^{2}+\frac{2 \mu_{\sigma}}{3}
\end{array}\right], \\
& \tau_{n}=\sqrt{\sigma_{n s}^{2}+\sigma_{n d}^{2}}
\end{aligned}
$$

при

$\sigma_{n i}=\tau\left[\left(1-\mu_{\sigma}\right) l_{1 n} l_{1 i}-\left(1+\mu_{\sigma}\right) l_{3 n} l_{3 i}\right], i=s, d$,

где $\sigma_{n s}$ и $\sigma_{n d}$ - компоненты касательных напряжений на плоскости разрыва (очаг) в направлении его простирания (strike) и погружения (dip), $l_{j i}-$ направляющие косинусы главных напряжений $\sigma_{j}$ $(j=1,2,3)$ с соответствующим вектором $(\boldsymbol{n}, \boldsymbol{s}, \boldsymbol{d})$.

\section{6. РЕЗУЛЬТАТЫ}

Обработка исходных сейсмологических данных производилась в длиннопериодном режиме (среднее по всему периоду времени) реконструкции 
[Rebetsky, 2003] в узлах сетки $0.5 \times 0.5$ и глубиной 10 км (приходится максимум событий). Узлы сетки, в которых получены данные о напряжениях, отвечали центрам формирования однородных выборок механизмов очагов землетрясений.

Результатом реконструкции являются данные об ориентации трех главных осей тензора напряжений и приращений сейсмотектонических деформаций, а также данные о коэффициенте Лоде-Надаи этих тензоров. По результатам расчетов были получены данные о напряжениях в 160 доменах земной коры. Их распределение неравномерное, большая часть приходится на кору Апеннин, Динарид, о. Сицилия, Альп, Адриатического моря, островов Корфу, Кефалония, Закинф. В настоящей работе были реализованы два первых этапа МКА, позволяющие получать данные о форме и направлениях ориентации главных осей эллипсоида напряжений (главные оси напряжений), данные о геодинамическом режиме, коэффициенте Лоде - Надаи и данные о поддвиговых касательных напряжениях.

В рамках МКА знаки напряжений определяются так, как это принято в классической механике. Растягивающие напряжения положительные. Поскольку оси алгебраически максимальных главных напряжений определяют и направление максимального девиаторного растяжения, далее их будем именовать наибольшим растяжением или просто растяжением. Оси алгебраически минимального главного напряжения будем именовать как наибольшее сжатие или просто сжатие.

В коре Апеннин мы наблюдаем ярко выраженную субгоризонтальную ориентацию осей максимального растяжения перпендикулярно линиям разломов (рис. 4). При этом вдоль западного побережья они полого погружаются на запад - юго-запад, а вдоль восточного - на восток - северо-восток. Оси максимального сжатия субвертикальны везде, кроме восточной части и севера Апеннин и долины реки По. Таким образом, в коре западных и центральных Апеннин разломы должны отвечать сбросовой кинематике.

Напряженное состояние в коре района долины реки По сходно с напряженным состоянием коры и Адриатического моря. Здесь оси растяжения субвертикальны, а максимального сжатия - субгоризонтальны и ориентированы в основном в северовосточном направлении. При этом при переходе к коре Динарид и южных Альп происходит постепенный разворот осей в меридиональном и даже север - северо-западном направлении. Таким образом, оси максимального сжатия практически везде ортогональны главным хребтам горных сооружений. Согласно обобщению роза-диаграмм, в исследуемом регионе преобладает либо субгоризонтальная, либо субвертикальная ориентация растяже- ний, с направлением погружений на $\mathrm{CB}, \mathrm{B}$ и Ю3, а для осей сжатий в пологое погружение - с направлениями на ССЗ и ЮЗ.

Из взаимоотношения ориентации осей главных напряжений с вектором на зенит следует, что в коре Апеннин основным типом геодинамического режима является горизонтальное растяжение (рис. $5, a$ ). Южное и северное ограничение этого режима представлено горизонтальным сжатием в коре долины реки По, а также горизонтальным сдвигом и сжатием в коре Сицилии и Калабрийской дуге. В коре Адриатического моря имеет место режим горизонтального сжатия, который в локальных участках коры Динарид трансформируется в режимы горизонтального сдвига и даже растяжения.

Соотношение главных напряжений определяется значением коэффициента Лоде - Надаи. Там, где этот коэффициент близок к -1 (одноосное растяжение), алгебраически максимальные напряжения близки к промежуточному главному напряжению. Там, где этот коэффициент близок к +1 (одноосное сжатие), к промежуточному главному напряжению близки алгебраически минимальные главные напряжения.

Из результатов реконструкции видно, что существует достаточно ограниченное число областей с состояниями, близкими к одноосному сжатию или растяжению (рис. 5, б). В коре Динарид больше областей одноосного сжатия и сочетание этого вида тензора напряжений с чистым сдвигом. В то же время в коре Апеннин и о. Сицилия больше областей одноосного растяжения и сочетания этого вида тензора напряжений с чистым сдвигом.

В основном в коре исследуемого региона реализуется напряженное состояние с видом тензора, отвечающего чистому сдвигу. Домены с этим типом тензора в коре Апеннин стремятся занять его осевую часть. В коре Динарид они, наоборот, тяготеют к его западным окраинам. Кора Адриатического моря практически полностью отвечает чистому сдвигу.

Обобщая результаты анализа параметров напряженного состояния, отметим, что напряженное состояние коры Адриатики и западных Динарид соответствует состоянию коры субдукционных зон [Rebetsky, 2009; Rebetsky, Polets, 2014; Rebetsky et al., 2016]. Состояние коры Апеннин соответствует напряженному состоянию коры крупных платообразных поднятий Центральной Азии и Тибета [Rebetsky, Alekseev, 2014; Rebetsky, 2015].

Важным элементом в исследовании механизма формирования режимов растяжения и сжатия в коре является характер распределения и направления вектора касательных напряжений, возникающих на границе контакта коры и мантии $\left(\boldsymbol{\tau}_{z}\right)$, которые именуются как поддвиговые: 


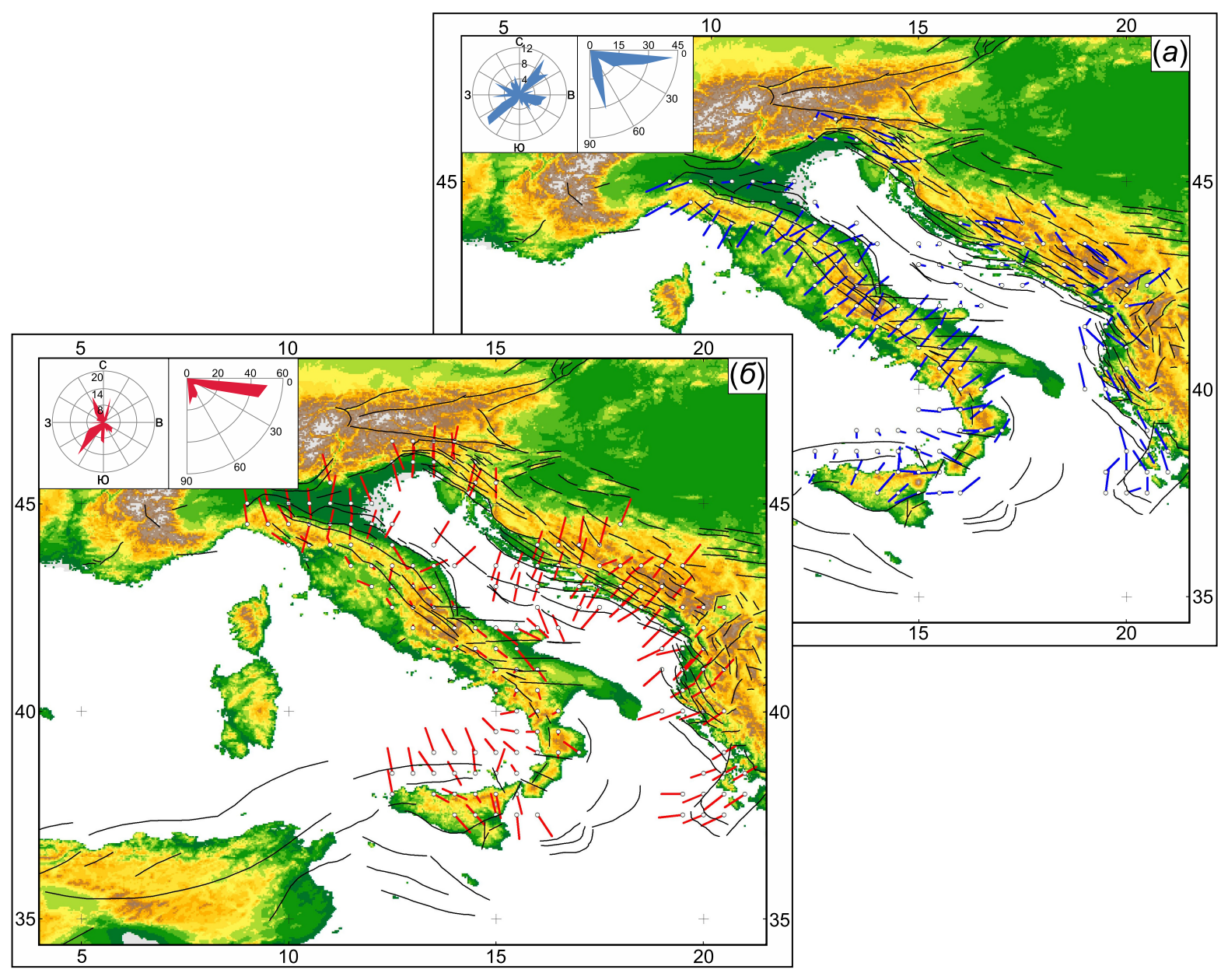

Рис. 4. Проекции на горизонтальную плоскость осей погружения алгебраически максимальных $\sigma_{1}(a)$ и минимальных $\sigma_{3}($ (б) главных напряжений. В нижнем левом углу показаны роза-диаграммы простираний и углов погружения соответствующих осей главных напряжений. Кружок определяет начало вектора погружения, а его длина зависит от крутизны погружения оси главного напряжения. Если угол погружения составлял менее $7.5^{\circ}$, то эта ось изображалась в виде вектора с кружком в середине. Линии серого цвета - разломы земной коры (SHARE, The European Database of Seismogenic Faults, http://diss.rm.ingv.it/share-edsf/SHARE_WP3.2_Downloads.html).

Fig. 4. Projections to the horizontal plane of the dip axes of algebraically maximum $(a)$ and minimum $\sigma_{3}(\sigma)$ principal stresses. Lower left corner - rose diagrams of the strikes and dip angles of the corresponding principal stress axes. Circle beginning of the sinking vector; the vector length depends on the steepness of dipping of the principal stress axis. For the dip angles less than $7.5^{\circ}$, the axes are shown as vectors with circles in the middle. Grey lines - faults in the crust (SHARE, The European Database of Seismogenic Faults, http://diss.rm.ingv.it/share-edsf/SHARE_WP3.2_Downloads.html).

$$
\boldsymbol{\tau}_{z}=\sigma_{z E} \boldsymbol{e}_{E}+\sigma_{z N} \boldsymbol{e}_{N}
$$

при

$$
\sigma_{z i}=\tau\left[\left(1-\mu_{\sigma}\right) l_{1 z} l_{1 i}-\left(1+\mu_{\sigma}\right) l_{3 z} l_{3 i}\right], i=N, E \text {, }
$$

где $\sigma_{z E}$ и $\sigma_{z N}-$ компоненты касательных напряжений в направлении на восток и север, действующие на горизонтальных площадках с нормалью вниз, $\boldsymbol{e}_{E}$ и $\boldsymbol{e}_{N}$ - единичные орты в направлении на восток и север, $l_{1 z}$ - направляющие косинусы главных напряжений в топоцентрической системе координат.

На рис. 6 показана ориентация и величины таких касательных напряжений, нормированных на значения максимальных касательных напряжений. Максимальные значения этого отношения, близкие к единице, отражают тот факт, что на данном участке коры ее подошва является плоскостью действия максимальных касательных напряжений.

В коре Альп преобладает направление поддвиговых касательных напряжений на С3 при высоком уровне их интенсивности. Это фактически означает, что мантия сопротивляется растеканию Альп на ЮВ. Существует также достаточно выдержанная южная и юго-западная ориентация этих напряжений в коре западной части Апеннин при среднем уровне их значений. Обратим внимание, что в восточной части коры этой части Апеннин нет столь 


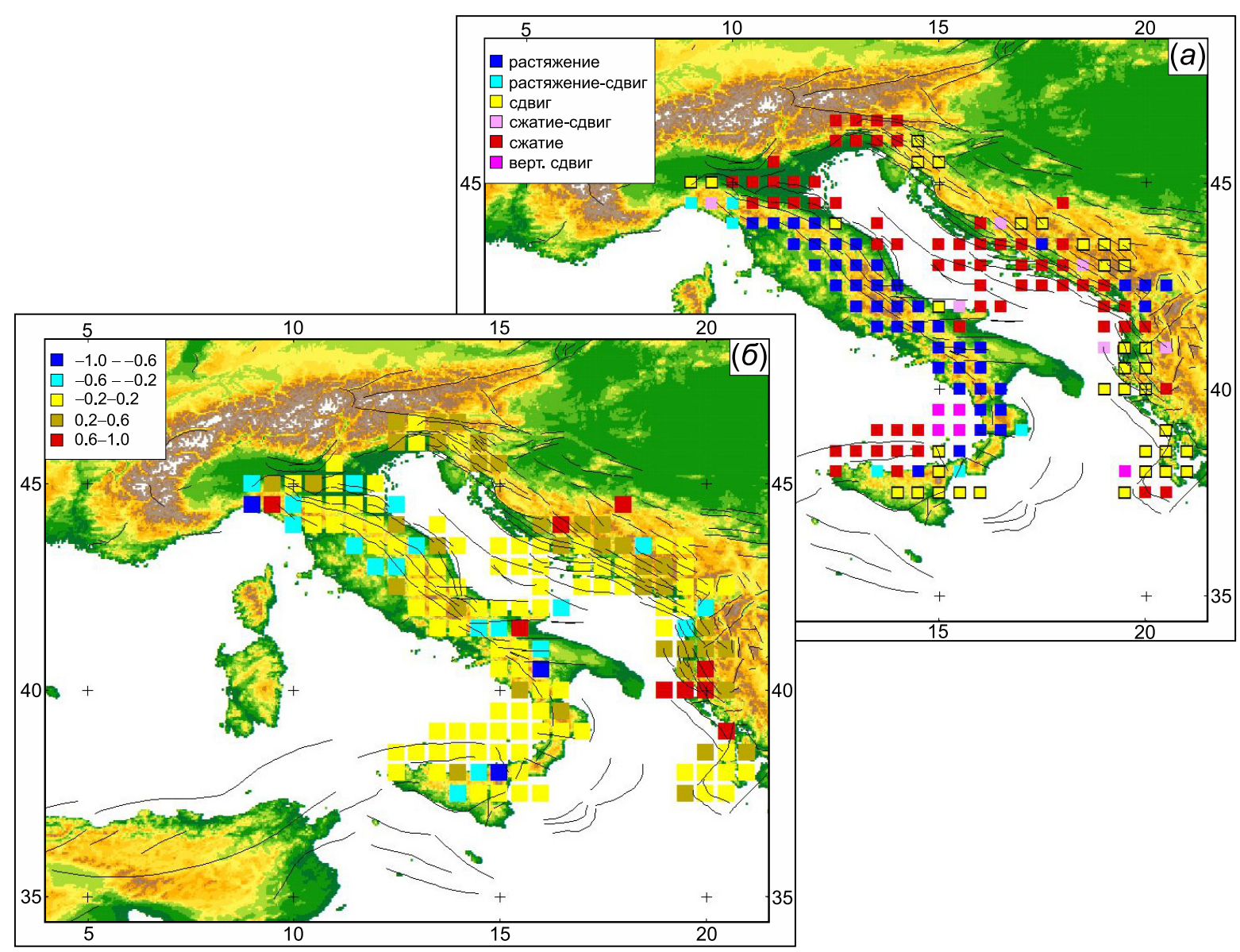

Рис. 5. Геодинамический режим напряженного состояния $(a)$ и параметр эллипсоида напряжений - коэффициент Лоде - Надаи (б). На диаграмме вверху справа показана представительность каждого из геодинамических режимов.

Fig. 5. Geodynamic regimes of the stress state (a), and the parameter of the stress ellipsoid (Lode - Nadai coefficient) (б). The diagram (top right) shows the representativeness of each geodynamic regime.

единой ориентации поддвиговых напряжений. Выявленная единая ориентация осей поддвиговых касательных напряжений сменяется на почти прямо противоположную вблизи $14^{\circ}$ в.д. Далее к югу происходит удивительно согласованный разворот ориентации поддвиговых касательных напряжений от моря к континенту, повторяя форму дуги береговой линии. Также в районе о. Закинф и о. Кефалония напряжения меняют свое направление с СВ на С. Поддвиговые касательные напряжения, близкие к максимальным, присутствуют в Дринском заливе и имеют направление на восток.

В коре Северных Динарид нет определенного направления касательных напряжений, структура мозаична. Ориентация этих напряжений становится согласованной к востоку от $18^{\circ}$ в.д. Здесь эти напряжения действуют субширотно от морской части коры к континентальной.

Анализ распределения в коре исследуемого региона эффективного давления (тектоническое давление минус давление флюида в трещинно-поровом пространстве) показывает его существенную неоднородность (рис. 6, б). Здесь можно выделить участки среднего, низкого и высокого уровня эффективного давления и соответственно (рис. 6, врезка) максимальных касательных напряжений. Линейные размеры этих областей оцениваются в 150-200 км. Поскольку основная система разломов как для Апеннинского полуострова, так и для Динарид имеет северо-западное простирание, вдоль разломов выделяются участки повышенного и пониженного уровня напряжений. При этом большие перепады в напряжениях свойственны разломам Динарид. Центральная сеть разломов Апеннин в целом находится в поле с более высоким уровнем напряжений.

\section{7. ОБСУЖДЕНИЕ}

Полученная по результатам реконструкции ориентация осей главных напряжений с одной стороны вполне соответствует представлениям о сближении Африканской и Евразийской плит и субдук- 


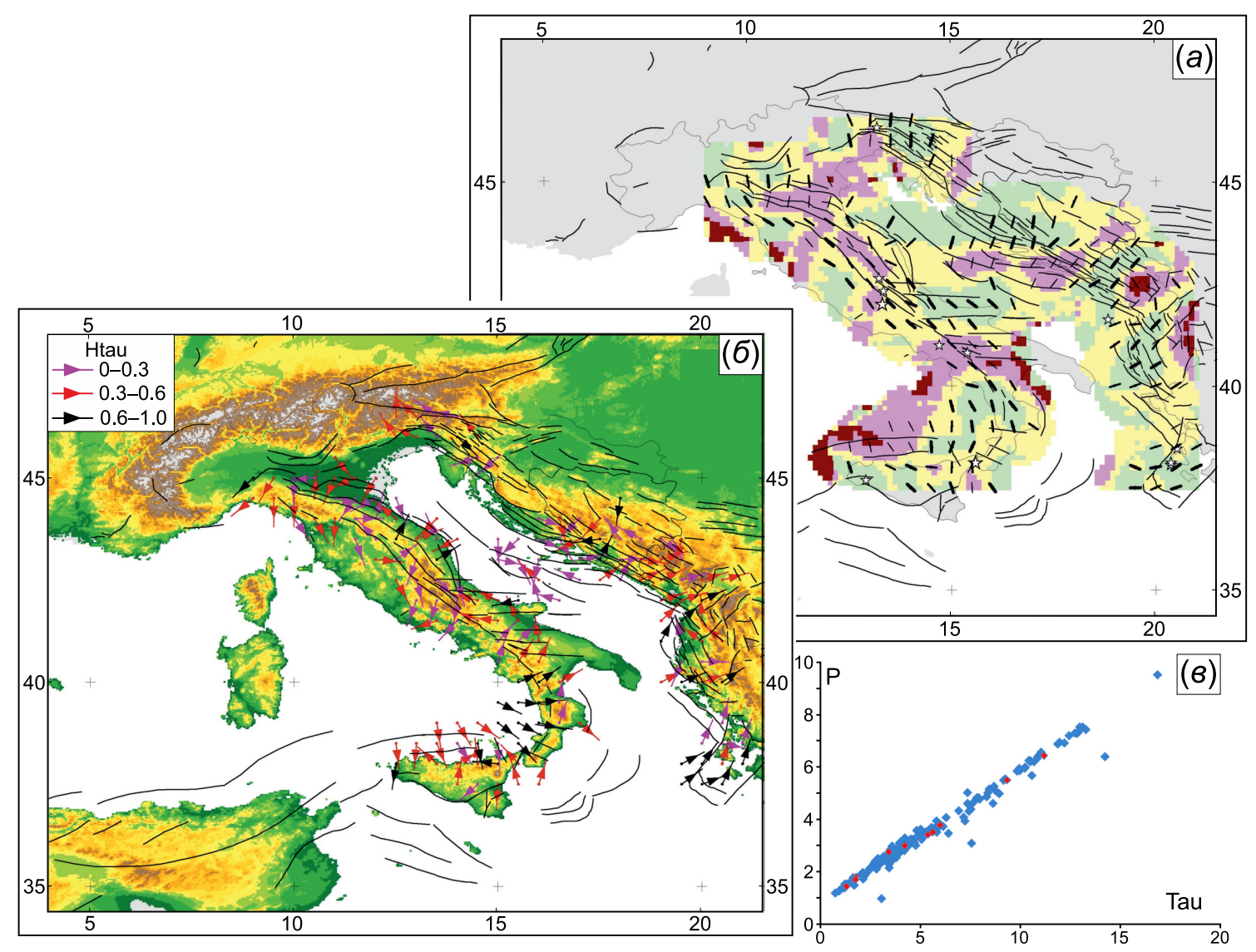

Рис. 6. Поддвиговые касательные напряжения, действующие на горизонтальных площадках с нормалями вниз (б), относительные величины напряжений, действующих со стороны мантии на кору (звездочками отмечены наиболее сильные события М>6) (a), зависимость эффективного давления от относительных величин напряжений ( 6$)$.

Fig. 6. Underthrust stresses on horizontal planes with downward normal (б); values of relative stresses acting from the mantle to the crust (asterisks - strongest seismic events, $\mathrm{M}>6)(a)$; effective pressure as a function of relative stress values $(b)$.

ции Адриатической плиты под Динариды. Определенные противоречия с этим вызывает режим горизонтального растяжения в коре Апеннин. В случае, если активным механизмом нагружения литосферы исследуемого региона является СВ движение Африканской плиты, сжатие должно нарастать с ЮЗ на СВ. Однако из данных об ориентации осей главных напряжений (см. рис. 3) видно, что в самой южной части исследуемого региона при движении с юго-запада на северо-восток в СВ направлении действуют сначала оси минимального сжатия $\sigma_{1}$ (кора Сицилии), далее в этом направлении действуют уже оси промежуточного главного напряжения G2 (кора юга Апеннин) и только в коре южных Динарид в этом направлении начинают действовать напряжения максимального сжатия. Такая же последовательная смена главных напряжений наблюдается также несколько севернее от коры Апеннин к коре Динарид. В центральной и северной части исследуемого региона наблюдается резкая смена индекса главных напряжений в СВ направлении от минимального сжатия $\sigma_{1}$ к максимальному сжатию $\sigma_{3}$ без наличия участков, где в этом направлении действовали бы оси промежуточного главного напряжения $\sigma_{2}$.

Против субдукции Африканской плиты (океанской ее части) под Евразийскую также свидетельствует отсутствие однонаправленного действия в CB направлении поддвиговых касательных напряжений (рис. 6, a). Такая закономерность отвечает всем современным зонам субдукции Тихого, Индийского и Атлантического океанов [Rebetsky, Polets, 2014; Rebetskii, Marinin, 2006; Rebetsky, 2009].

Во всех случаях горизонтальное сжатие уменьшается с северо-востока на юго-запад. Согласно такой закономерности скорее можно говорить о давлении Евразийской плиты на Средиземноморскую литосферную плиту. В пользу этого вывода говорит и СВ направление поддвиговых касательных напряжений в южной части коры Динарид (рис. 6, a).

Другим вариантом интерпретации результатов реконструкции напряжений являются представления о мелкомасштабной конвекции в верхней мантии. При этом восходящая ветвь конвекции находится под центральной частью Апеннин, а нисходящая - под Динаридами. Вероятно, более выра- 
P.A. Savvichev, Yu.L. Rebetsky: Modern state of crustal stresses of the Apennine Peninsula and adjacent territories...

женно этот процесс идет в южной части исследуемого региона. Слабовыраженный горный рельеф в осевой зоне Апеннин, возможно, связан также с механизмом гравитационного всплывания коры.

Анализ распределения сейсмических событий по отношению к полю значений эффективного давления (рис. 6, б) показывает, что наиболее сильные события в последнее время (Норча, 2016 г., $M_{\mathrm{w}}=6.3$; Л'Акуила, 2009 г., $M_{\mathrm{w}}=6.3$ и др.) происходили в областях с минимальными и средними относительными величинами напряжений. В то же время землетрясения меньших магнитуд могут попадать в области разного уровня напряжений. Это говорит о взаимосвязи масштабного уровня реконструированных напряжений только с сильными землетрясениями, размер очага которых превышает 10-20 км.

Об опасности участков низкого уровня эффективного давления для формирования сильных землетрясений говорилось в работах [Rebetsky, 2007a, 2007b; Rebetsky, Tatevossian, 2013]. Об этом говорят и современные исследования характера трения в очаговой области в рамках теории Rate\&State [Rice et al., 2001]. По результатам численного моделирования установлено, что хрупкому разрушению всегда предшествует этап разупрочнения породы [Rice et al., 2001; Kocharyan, 2014], приводящий к снижению уровня напряжений (давления и девиаторных напряжений). После формирования на разрыве участка пониженной прочности и происходит хрупкое разрушение.

\section{8.ЗАКЛЮЧЕНИЕ}

Одной из задач, поставленных в данном исследовании, было сравнение наших данных о реконструкции и результатов, полученных с использованием метода М.Л. Зобак для коры Италии (см. рис. 1). Ориентации осей наибольшего горизонтального сжатия имеют незначительные отклонения (менее $15^{\circ}$ ) от результатов М.Л. Зобак в доменах, где коэффициент Лоде - Надаи близок к нулю. Однако там, где вид эллипсоида напряжений принимает свои критические значения, равные +1 и -1 (одноосное сжатие и одноосное растяжение соответственно), ориентация существенно отличается и различие может быть до $45^{\circ}$, что подтверждает необходимость принимать во внимание вид эллипсоида напряжений при проведении реконструкции.

Распределение типов геодинамического режима говорит о преобладании горизонтального растяжения в коре Апеннин и горизонтального сжатия в коре Динарид. Режим сдвига и сжатия также наблюдается на дуге Калабрии около о. Сицилия. В долине реки По происходит резкая смена геодинамического режима с горизонтального растяжения в коре Апеннин на горизонтальное сжатие по границе разлома.

Закономерность распределения ориентаций поддвиговых касательных напряжений, действующих со стороны мантии на кору, не противоречит современным зонам субдукции. Около о. Сицилия происходит согласованный разворот ориентации поддвиговых касательных напряжений от моря к континенту, повторяя форму дуги береговой линии.

Формирование наиболее сильных событий $(\mathrm{M}>6)$ происходило в областях с минимальными и средними относительными величинами напряжений.

Полученная в результате реконструкции ориентация главных осей напряжений соответствует модели сближения тектонических плит. Наличие некоторых неоднородностей говорит о том, что на этих участках необходимо более детальное исследование.

Проведенные исследования показали, что современное напряженное состояние коры Апеннинского полуострова не связано с каким-то одним фактором внешнего воздействия и не может быть описано с помощью одной модели. Необходим комплексный подход и объединение трех деформационных механизмов, влияющих на данную структуpy.

\section{9. БЛАГОДАРНОСТИ}

Данная работа выполнена в рамках Госзаказа ИФЗ РАН.

\section{0. ЛИTEPATУPA / REFERENCES}

Bell J.S., 1996. In situ stresses in sedimentary rocks (part 2): applications of stress measurements. Geoscience Canada 23 (3), 135-153.

Boccaletti M., Conedera C., Dainelli P., Gočev P., 1982. The recent (Miocene-Quaternary) regmatic system of the Western Mediterranean region: a new model of ensialic geodynamic evolution, in a context of plastic/rigid deformation. Journal of Petroleum Geology 5 (1), 31-49. https://doi.org/10.1111/j.1747-5457.1982.tb00559.x.

Carminati E., Scrocca D., Doglioni C., 2010. Compaction-induced stress variations with depth in an active anticline: Northern Apennines, Italy. Journal of Geophysical Research: Solid Earth 115 (B2), B02401. https://doi.org/ 10.1029/2009JB006395. 
Channell J.E.T., 1986. Palaeomagnetism and continental collision in the Alpine belt and the formation of late-tectonic extensional basins. In: M.P. Coward, A.C. Reis (Eds.), Collision tectonics. Geological Society, London, Special Publications, vol. 19, p. 261-284. https://doi.org/10.1144/GSL.SP.1986.019.01.15.

Coblentz D.D., Richardson R.M., 1995. Statistical trends in the intraplate stress field. Journal of Geophysical Research: Solid Earth 100 (B10), 20245-20255. https://doi.org/10.1029/95JB02160.

Faccenna C., Davy P., Brun J.P., Funiciello R., Giardini D., Mattei M., Nalpas T., 1996. The dynamics of back-arc extension: an experimental approach to the opening of the Tyrrhenian Sea. Geophysical Journal International 126 (3), 781-795. https://doi.org/10.1111/j.1365-246X.1996.tb04702.x.

Heidbach O., Rajabi M., Reiter K., Ziegler M., WSM Team, 2016. World Stress Map Database Release 2016. GFZ Data Services. https://doi.org/10.5880/WSM.2016.001.

Heidbach O., Reinecker J., Tingay M., Müller B., Sperner B., Fuchs K., Wenzel F., 2007. Plate boundary forces are not enough: Second-and third-order stress patterns highlighted in the World Stress Map database. Tectonics 26 (6), TC6014. https://doi.org/10.1029/2007TC002133.

Heidbach O., Tingay M., Barth A., Reinecker J., Kurfeß D., Müller B., 2008. The World Stress Map database release. The World Stress Map Project. https://doi.org/10.1594/GFZ.WSM.Rel2008.

Heidbach O., Tingay M., Barth A., Reinecker J., Kurfeß D., Müller B., 2010. Global crustal stress pattern based on the World Stress Map database release 2008. Tectonophysics 482 (1-4), 3-15. https://doi.org/10.1016/j.tecto.2009. 07.023.

Hillis R.R., Reynolds S.D., 2000. The Australian stress map. Journal of the Geological Society 157 (5), $915-921$. https://doi.org/10.1144/jgs.157.5.915.

Horvath F., Berckhemer H., 1982. Mediterranean back arc basins. In: H. Berckhemer, K. Hsü (Eds.), Alpine-Mediterranean geodynamics. AGU Geodynamics Series, vol. 7, p. 141-173. https://doi.org/10.1029/GD007p0141.

Kocharyan G.G., 2014. Scale effect in seismotectonics. Geodynamics \& Tectonophysics 5 (2), 353-385 (in Russian) [Кочарян Г.Г. Масштабный эффект в сейсмотектонике // Геодинамика и тектонофизика. 2014. Т. 5 . № 2. C. 353-385]. https://doi.org/10.5800/GT-2014-5-2-0133.

Malinverno A., Ryan W.B., 1986. Extension in the Tyrrhenian Sea and shortening in the Apennines as result of arc migration driven by sinking of the lithosphere. Tectonics 5 (2), 227-245. https://doi.org/10.1029/TC005i002p 00227.

Mantovani E., Albarello D., Babucci D., Tamburelli C., 1993. Post-tortonian deformation pattern in the Central Mediterranean: a result of extrusion tectonics driven by the Africa-Eurasia convergence. In: E. Boschi, E. Mantovani, A. Morelli (Eds.), Recent evolution and seismicity of the Mediterranean region. Kluwer, Dordrecht, p. 65-104. https://doi.org/10.1007/978-94-011-2016-6_3.

Mariucci M., Amato A., Gambini R., Giorgioni M., Montone P., 2002. Along-depth stress rotations and active faults: an example in a 5-km deep well of Southern Italy. Tectonics 21 (4), 1021. https://doi.org/10.1029/2001TC001338.

Müller B., Zoback M.L., Fuchs K., Mastin L., Gregersen S., Pavoni N., Stephansson O., Ljunggren C., 1992. Regional patterns of tectonic stress in Europe. Journal of Geophysical Research: Solid Earth 97 (B8), 11783-11803. https://doi.org/ 10.1029/91JB01096.

Patacca E., Scandone P., 1989. Post-Tortonian mountain building in the Apennines. The role of the passive sinking of a relic lithospheric slab. In: A. Boriani, M. Bonafede, G.B. Piccardo, G.B. Vai (Eds.), The lithosphere in Italy. Advances in Earth science research, vol. 80, p. 157-176.

Patacca E., Sartori R., Scandone P., 1990. Tyrrhenian basin and Apenninic arcs: kinematic relations since Late Tortonian times. In: Memorie della Societa Geologica Italiana, vol. 45, p. 425-451.

Pondrelli S., Salimbeni S., Ekström G., Morelli A., Gasperini P., Vannucci G., 2006. The Italian CMT dataset from 1977 to the present. Physics of the Earth and Planetary Interiors 159 (3-4), 286-303. https://doi.org/10.1016/j.pepi. 2006.07.008.

Rebetskii Y.L., Marinin A.V., 2006. Stressed state of the Earth's crust in the western region of the Sunda subduction zone before the Sumatra-Andaman earthquake on December 26, 2004. Doklady Earth Sciences 407 (1), 321-325. https://doi.org/10.1134/S1028334X06020383.

Rebetsky Y.L., 2003. Stress-strain State and Mechanical Properties of Natural Massifs According to the Data on the Earthquake Focal Mechanisms and Structural-Kinematic Characteristics of Fractures. PhD Thesis (Doctor of Physics and Mathematics). UIPE RAS, Moscow, 455 p. (in Russian) [Ребецкий Ю.Л. Напряженно-деформированное состояние и механические свойства природных массивов по данным о механизмах очагов землетрясений и структурно-кинематическим характеристикам трещин: Дис. ... докт. физ.-мат. наук. М.: ОИФЗ РАН, 2003. 455 c.].

Rebetsky Y.L., 2007a. New data on natural stresses in the preparation area of a strong earthquake. The model of earthquake source. Geophysical Journal 29 (6), 92-110 (in Russian) [Ребецкий Ю.Л. Новые данные о природных напряжениях в области подготовки сильного землетрясения. Модель очага землетрясения // Геофизический журнал. 2007. Т. 29. № 6. С. 92-110].

Rebetsky Y.L., 2007b. Tectonic stresses and areas of the trigger mechanism of earthquake occurrence. Fizicheskaya Mezomekhanika (Physical Mesomechanics) 10 (1), 25-37 (in Russian) [Ребецкий Ю.Л. Тектонические напряже- 
ния и области триггерного механизма возникновения землетрясений // Физическая мезомеханика. 2007. T. 10. № 1. C. 25-37].

Rebetsky Y.L., 2009. Stress state of the Earth's crust of the Kuril Islands and Kamchatka before the Simushir earthquake. Russian Journal of Pacific Geology 3 (5), 477-490. https://doi.org/10.1134/S1819714009050108.

Rebetsky Y.L., 2015. On the specific state of crustal stresses in intracontinental orogens. Geodynamics \& Tectonophysics 6 (4), 437-466 (in Russian) [Ребецкий Ю.Л. Об особенности напряженного состояния коры внутриконтинентальных орогенов // Геодинамика и тектонофизика. 2015. Т. 6. № 4. C. 437-466]. https://doi.org/10.5800/ GT-2015-6-4-0189.

Rebetsky Y.L., Alekseev R.S., 2014. The field of recent tectonic stresses in Central and South-Eastern Asia. Geodynamics \& Tectonophysics 5 (1), 257-290 (in Russian) [Ребецкий Ю.Л., Алексеев Р.С. Поле современных тектонических напряжений Средней и Юго-Восточной Азии // Геодинамика и тектонофизика. 2014. Т. 5. № 1. С. 257-290]. https://doi.org/10.5800/GT-2014-5-1-0127.

Rebetsky Y.L., Kuchai O.A., Marinin A.V., 2013. Stress state and deformation of the Earth's crust in the Altai-Sayan mountain region. Russian Geology and Geophysics 54 (2), 206-222. https://doi.org/10.1016/j.rgg.2013.01.011.

Rebetsky Y.L., Kuchai O.A., Sycheva N.A., Tatevossian R.E., 2012. Development of inversion methods on fault slip data: Stress state in orogenes of the Central Asia. Tectonophysics 581, 114-131. https://doi.org/10.1016/j.tecto.2012. 09.027.

Rebetsky Y.L., Kuzikov S.I., 2016. Active faults of the northern Tien Shan: tectonophysical zoning of seismic risk. Russian Geology and Geophysics 57 (6), 967-983. https://doi.org/10.1016/j.rgg.2016.05.004.

Rebetsky Y.L., Lermontova A.S., 2016. Registration of supercritical conditions of geologic environment and challenges in earthquake source remote sensing. Bulletin of Kamchatka Regional Association Educational-Scientific Center. Earth Sciences (4), 115-123 (in Russian) [Ребецкий Ю.Л., Лермонтова А.С. Учет закритического состояния геосреды и проблема дальнодействующего влияния очагов землетрясений // Вестник КРАУНЦ. Серия: Науки о Земле. 2016. № 4. С. 115-123].

Rebetsky Y.L., Polets A.Y., 2014. The state of stresses of the lithosphere in Japan before the catastrophic Tohoku earthquake of 11 March 2011. Geodynamics \& Tectonophysics 5 (2), 469-506 (in Russian) [Ребецкий Ю.Л., Полец А.Ю. Напряженное состояние литосферы Японии перед катастрофическим землетрясением Тохоку 11.03.2011 // Геодинамика и тектонофизика. 2014. T. 5. № 2. С. 469-506]. https://doi.org/10.5800/GT-2014-5-2-0137.

Rebetsky Y.L., Polets A.Y., Zlobin T.K., 2016. The state of stress in the Earth's crust along the northwestern flank of the Pacific seismic focal zone before the Tohoku earthquake of 11 March 2011. Tectonophysics 685, 60-76. https://doi.org/10.1016/j.tecto.2016.07.016.

Rebetsky Y.L., Tatevossian R.E., 2013. Rupture propagation in strong earthquake sources and tectonic stress field. Bulletin de la Société Géologique de France 184 (4-5), 335-346. https://doi.org/10.2113/gssgfbull.184.4-5.335.

Reutter K.J., Giese P., Closs H., 1980. Lithospheric split in the descending plate: observations from the Northern Apennines. Tectonophysics 64 (1-2), T1-T9. https://doi.org/10.1016/0040-1951(80)90254-1.

Rice J.R., Lapusta N., Ranjith K., 2001. Rate and state dependent friction and the stability of sliding between elastically deformable solids. Journal of the Mechanics and Physics of Solids 49 (9), 1865-1898. https://doi.org/10.1016/ S0022-5096(01)00042-4.

Royden L.H., 1993. Evolution of retreating subduction boundaries formed during continental collision. Tectonics 12 (3), 629-638. https://doi.org/10.1029/92TC02641.

Royden L., Patacca E., Scandone P., 1987. Segmentation and configuration of subducted lithosphere in Italy: An important control on thrust-belt and foredeep-basin evolution. Geology 15 (8), 714-717. https://doi.org/10.1130/ 0091-7613(1987)15<714:SACOSL>2.0.CO;2.

Sassi W., Faure J.L., 1996. Role of faults and layer interfaces on the spatial variation of stress regimes in basins: inferences from numerical modelling. Tectonophysics 266 (1-4), 101-119. https://doi.org/10.1016/S00401951(96)00185-0.

Suppe J., 2007. Absolute fault and crustal strength from wedge tapers. Geology 35 (12), 1127-1130. https://doi.org/ 10.1130/G24053A.1.

Tapponnier P., 1977. Evolution tectonique du systeme alpin en Mediterranee; poinconnement et ecrasement rigideplastique. Bulletin de la Société géologique de France 7 (3), 437-460. https://doi.org/10.2113/gssgfbull.S7XIX.3.437.

Tingay M.R., Hillis R.R., Morley C.K., Swarbrick R.E., Drake S.J., 2005. Present-day stress orientation in Brunei: a snapshot of 'prograding tectonics' in a Tertiary delta. Journal of the Geological Society 162 (1), 39-49. https://doi.org/ 10.1144/0016-764904-017.

Tingay M., Morley C., King R., Hillis R., Coblentz D., Hall R., 2010. Present-day stress field of Southeast Asia. Tectonophysics 482 (1-4), 92-104. https://doi.org/10.1016/j.tecto.2009.06.019.

Yale D.P., 2003. Fault and stress magnitude controls on variations in the orientation of in situ stress. In: M. Ameen (Ed.), Fracture and in-situ stress characterization of hydrocarbon reservoirs. Geological Society, London, Special Publications, vol. 209, p. 55-64. https://doi.org/10.1144/GSL.SP.2003.209.01.06. 
Zoback M.L., 1992. First-and second-order patterns of stress in the lithosphere: The World Stress Map Project. Journal of Geophysical Research: Solid Earth 97 (B8), 11703-11728. https://doi.org/10.1029/92JB00132.

Zoback M.L., Zoback M.D., Adams J., Assumpção M., Bell S., Bergman E.A., Blümling P., Brereton N.R., Denham D., Ding J., Fuchs K., Gay N., Gregersen S., Gupta H.K., Gvishiani A., Jacob K., Klein R., Knoll P., Magee M., Mercier J.L., Müller B.C., Paquin C., Rajendran K., Stephansson O., Suarez G., Suter M., Udias A., Xu Z.H., Zhizhin M., 1989. Global patterns of tectonic stress. Nature 341 (6240), 291-298. https://doi.org/10.1038/341291a0.

\section{Павел Александрович Саввичев}

М.H.C.

Институт физики Земли им. О.Ю. Шмидта РАН

123242, ГСП-5, Москва Д-242, ул. Большая Грузинская, 10, Россия

\ e-mail: psavvichev@gmail.com

\section{Юрий Леонидович Ребецкий}

докт. физ.-мат. наук, зав. лабораторией

Институт физики Земли им. О.Ю. Шмидта РАН

123242, ГСП-5, Москва Д-242, ул. Большая Грузинская, 10, Россия

e-mail: reb@ifz.ru
Pavel A. Savvichev

Junior Researcher

O.Yu. Schmidt Institute of Physics of the Earth of RAS

10 Bol'shaya Gruzinskaya street, Moscow D-242 123242, GSP-5, Russia

\section{Yuri L. Rebetsky}

Doctor of Physics and Mathematics, Head of Laboratory

O.Yu. Schmidt Institute of Physics of the Earth of RAS

10 Bol'shaya Gruzinskaya street, Moscow D-242 123242, GSP-5, Russia 